\title{
RECUPERAÇÃO DE COBRE DE LIXIVIADO DE REJEITO DE NÍQUEL UTILIZANDO RESINA QUELANTE*
}

\section{Resumo}

Amilton Barbosa Botelho Junior ${ }^{1}$ Monica Maria Jimenez Correa² Isadora Dias Perez ${ }^{3}$

Denise Crocce Romano Espinosa ${ }^{4}$ Jorge Alberto Soares Tenório 5

A busca por metais para atender demandas crescentes vem em contracorrente à diminuição dos teores nas reservas. Desta forma, a busca por novas rotas de extração de metais aliada ao desenvolvimento sustentável se faz cada vez mais necessário. $O$ presente trabalho teve por objetivo a recuperação de cobre do lixiviado proveniente de rejeito de níquel laterítico, que contém os seguintes metais: alumínio, cobalto, cromo, cobre, ferro, magnésio, manganês, níquel e zinco. Para isto, utilizou-se o processo de troca-iônica com a resina quelante Lewatit TP 207, uma resina catiônica com grupo funcional iminodiacetato. Estudou-se o tempo de reação e a influência de $\mathrm{pH}$. O tempo de reação foi variado entre 0 e 24 horas e o pH de 0.5 a 2. Não foi estudado maiores valores de $\mathrm{pH}$, pois haveria precipitação do ferro. A reação de adsorção pela resina entrou em equilíbrio em 180min. Em pH 2 teve maior recuperação de cobre, pois havia menor competição entre $\mathrm{H}^{+}$e os cátions metálicos do licor, onde foi possível recuperar $50 \%$ do cobre. Os ensaios ocorreram em batelada com agitação de 200rpm, e as análises foram feitas usando a técnica de fluorescência de raios $X$ por energia dispersiva (EDX).

Palavras-chave: Troca-iônica; Hidrometalurgia; Níquel Laterítico.

\section{RECOVERY OF COPPER FROM NICKEL WASTE LEACHING USING CHELATING} RESIN

\section{Abstract}

The search for metals to comply with increasing demand comes in countercurrent of decreasing of grade minerals. In this way, the search of new routes of extraction of metals allied sustainable development becoming increasingly necessary. The present work has as objective the recovery of copper from nickel laterite tailings leaching, that has the fallowing metals: aluminum, cobalt, chromium, copper, iron, magnesium, manganese, nickel and zinc. For this, it was used ion exchange process with chelating resin Lewatit TP 207, a cationic resin with iminodiacetate functional group. It was studied reaction time and $\mathrm{pH}$ influence. The reaction time was varied between 0 and 24 hours and $\mathrm{pH} 0.5-2$. It wasn't studied $\mathrm{pH}$ above 2 , because it would have iron precipitation. The adsorption reaction using chelating resin went into a balance in 180min. In pH 2 has more recovery of copper, because has less competition with $\mathrm{H}^{+}$and metals of the liquor, where it was possible recovery $50 \%$ of copper. The tests were realized in batch in 200rpm, and the analyzes were realized using X-ray fluorescence technique by dispersive energy (EDX).

Keywords: lon-exchange; Hydrometallurgy; Nickel Laterite

1 Engenheiro Químico, mestrando do Departamento de Engenharia Química, Escola Politécnica da Universidade de São Paulo, São Paulo, Brasil, amilton.junior@usp.br (autor para correspondência).

2 Engenheira Química, doutoranda do Departamento de Engenharia Química, Escola Politécnica da Universidade de São Paulo, São Paulo,Brasil, monicamariajimenezc@gmail.com.

3 Engenheira Ambiental mestrando do Departamento de Engenharia Química, Escola Politécnica da Universidade de São Paulo, São Paulo, Brasil, dpisadora@gmail.com.

4 Engenheira Metalurgista, doutora pelo Departamento de Materiais e Metalurgia, professora associada do Departamento de Engenharia Química, Escola Politécnica da Universidade de São Paulo, São Paulo,Brasil, espinosa@usp.br.

5 Engenheiro Metalurgista, doutor pelo Departamento de Materiais e Metalurgia, professor titular do Departamento de Engenharia Química, Escola Politécnica da Universidade de São Paulo, São Paulo, Brasil, jtenorio@usp.br 


\section{INTRODUÇÃO}

O minério de níquel pode ser encontrado em dois tipos de reservas: reservas sulfetadas e reservas lateríticas. A produção de níquel a partir de reservas lateríticas representa $40 \%$ do total, enquanto a quantidade de reservas são $70 \%$. Isto se deve pela complexidade que é para extrair níquel da reserva laterítica frente à sulfetada, além de demandar tratamentos complexos e onerosos, tornando o níquel mais caro do que de reservas sulfetadas, além de haver maior emissão de gases de efeito estufa $(1,2)$.

Com o aumento da demanda por metais como o níquel, e a diminuição dos teores das reservas (as reservas lateríticas há cerca de 1\% de níquel), o que dificulta ou torna o processo economicamente inviável, faz com que a busca por novas técnicas hidrometalúrgicas de extração, além de utilizar como reservas o que antes era considerado como rejeito, seja de interesse econômico. Outro ponto importante é que a reciclagem por si só não será o suficiente para atender a demanda mundial por metais (1-5).

Há um grande interesse da indústria mineradora brasileira em desenvolver técnicas de recuperação de metais de rejeitos, sobretudo após o ocorrido em novembro de 2015 na cidade de Mariana/MG (5-8). Entre as técnicas hidrometalúrgicas está a troca-iônica, que pode ser definido como o processo reversível de troca de íons entre a resina (insolúvel) e a solução. Para resinas catiônicas, os cátions da solução irão realizar o processo de troca com os cátions do grupo funcional da resina. Entre as vantagens desta técnica estão a separação quantitativas, aplicação em soluções com íons "traços" e até macroconcentrações, e versatilidade. Entre as aplicações, estão: recuperação de metais de rejeitos; amolecimento de água; e extração de urânio, ouro, prata, platina, entre outros (912).

O objetivo do trabalho foi utilizar a técnica de troca-iônica para recuperar cobre de um lixiviado proveniente de um rejeito de níquel, utilizando uma resina quelante. Analisou-se o tempo de reação entre 0 e 24 horas e a influência do $\mathrm{pH}$. Este último tem forte influência no processo de troca iônica, uma vez que o íon $\mathrm{H}^{+}$ compete com os cátions metálicos presentes na solução pelo sítio ativo da resina $(13,14)$.

\section{MATERIAIS E MÉTODOS}

\subsection{Pré-tratamento da resina}

A resina foi preparada (lavada) utilizando ácido clorídrico $4 \mathrm{~mol} . \mathrm{L}^{-1}$, em uma etapa, e hidróxido de sódio $2 \mathrm{~mol} . \mathrm{L}^{-1}$, em outra etapa, e água deionizada entre as etapas, a fim de realizar a remoção de impurezas. O procedimento foi realizado utilizando frascos erlenmeyer de $250 \mathrm{~mL}$, e agitação à $200 \mathrm{rpm}$ em $25^{\circ} \mathrm{C}$. Após o uso, a resina foi novamente lavada utilizando mesmo procedimento e com o auxílio de ultrassom.

\subsection{Preparo da solução sintética}

Para a realizar dos ensaios, preparou-se uma solução sintética a fim de simular o lixiviado do rejeito de níquel. Na Tabela 1 está apresentado a composição, em mg. $\mathrm{L}^{-1}$, dos metais do licor. Esta solução foi preparada a partir de sais de sulfato de cada metal. $\mathrm{O}$ pH foi então diminuído utilizando ácido sulfúrico concentrado até 
valor da solução real ( $\mathrm{pH}$ 0.5). A correção do pH foi realizada utilizando hidróxido de sódio $2 \mathrm{~mol} . \mathrm{L}^{-1}$.

Tabela 1: Composição do licor de níque

\begin{tabular}{llllllllll}
\hline \multirow{2}{*}{$\begin{array}{l}\text { Concentração } \\
\left.\text { (mg. } \text { L }^{-1}\right)\end{array}$} & $\mathbf{A l}$ & $\mathbf{C o}$ & $\mathbf{C r}$ & $\mathbf{C u}$ & $\mathbf{F e}$ & $\mathbf{M g}$ & $\mathrm{Mn}$ & $\mathbf{N i}$ & $\mathbf{Z n}$ \\
\cline { 2 - 9 } & 4101.5 & 78.1 & 195.2 & 146.9 & 18713.5 & 7774.5 & 397.2 & 2434.2 & 36.7 \\
\hline
\end{tabular}

Utilizou-se a resina quelante Lewatit TP 207, um tipo de resina capaz de formar ligações coordenadas com metais doadores de elétrons formando quelatos, tendo seletividade com alguns tipos de íons (11). Esta resina catiônica tem grupo funcional iminodiacetato, densidade de $1.17 \mathrm{~g} \cdot \mathrm{mL}^{-1}$, matriz de poliestireno em ligação cruzada e pH de trabalho entre 0 e 14. Os ensaios foram realizados em batelada, com $100 \mathrm{~mL}$ do liquor e $1 \mathrm{~mL}$ de resina em erlenmeyers de $250 \mathrm{~mL}$, em shaker orbital com velocidade de agitação de 200rpm.
A $q t=\left(C_{0}-C_{t}\right) x \frac{v}{m}$
(Equação 1 foi utilizada a fim de determinar a quantidade de íons em equilíbrio, onde $q_{t}$ é capacidade da adsorvida no equilíbrio, em massa de íon metálico por massa de resina $\left(\mathrm{mg}^{-g^{-1}}\right), \mathrm{C}_{0}$ e $\mathrm{C}_{t}$ são as concentrações dos íons no tempo 0 e no tempo $t\left(\mathrm{mg}^{\mathrm{L}} \mathrm{L}^{-1}\right)$, v é o volume do licor (L) e m é a massa da resina $(\mathrm{g})(13,15)$.

$$
\begin{gathered}
q_{t}=\left(C_{0}-C_{t}\right) x \frac{v}{m} \quad \text { (Equação 1) } \\
\text { A \%S }=\frac{\left(C_{0}-C_{t}\right)}{C_{0}} \times 100 \% \quad \text { (Equação } 2 \text { foi utilizada para determinar a }
\end{gathered}
$$
quantidade de íon adsorvido pela resina, em porcentagem (\%).

$$
\% S=\frac{\left(C_{0}-C_{t}\right)}{C_{0}} x 100 \% \quad \text { (Equação 2) }
$$

Outra forma de estudar a reação de troca-iônica é o cálculo do coeficiente de distribuição (Kd), que é calculado da seguite forma:

$$
K d=\frac{q_{e}}{C_{t}} x 1000 \quad \text { (Equação 3) }
$$

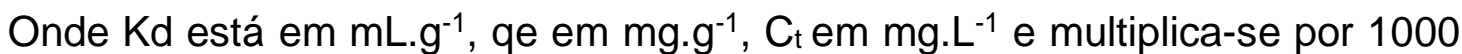
para converter $\mathrm{L}$ para $\mathrm{mL}$. Este coeficiente uma forma de medida direta da extensão da troca pelo qual o íon é removido da solução pela resina (12). Para análise de concentração, foi utilizado técnica de espectrometria de fluorescência de raios- $x$ de energia dispersiva (EDX), em um equipamento da marca PANalytical modelo Epsilon $3 X L$.

\section{RESULTADOS E DISCUSSÃO}

\subsection{Tempo de reação}

Os ensaios foram realizados a fim de determinar o tempo em que a reação de adsorção entra em equilíbrio. Os tempos de reação estudados foram: 0, 30, 60, 90, $120,180,240,300,480$ e 1440 (24 horas). Como é possível verificar na Figura 1, a reação entra em equilíbrio em 180min ( 3 horas). Os ensaios foram realizados em $\mathrm{pH}$ 0.5 . Para os ensaios variando o $\mathrm{pH}$, adotou-se como tempo de reação de $180 \mathrm{~min}$. 
Além do cobre, a capacidade de adsorção do ferro apresenta a mesma característica que a do cobre, entrando em equilíbrio em 180min (Figura 2).

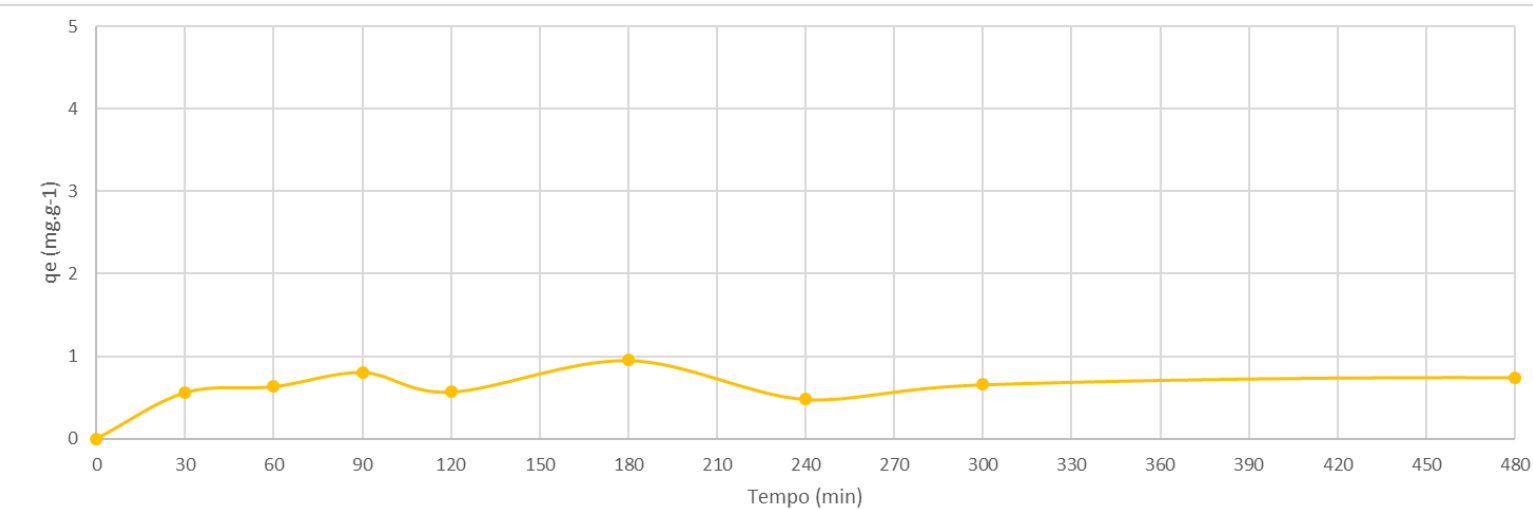

Figura 1: Variação de tempo na adsorção de cobre

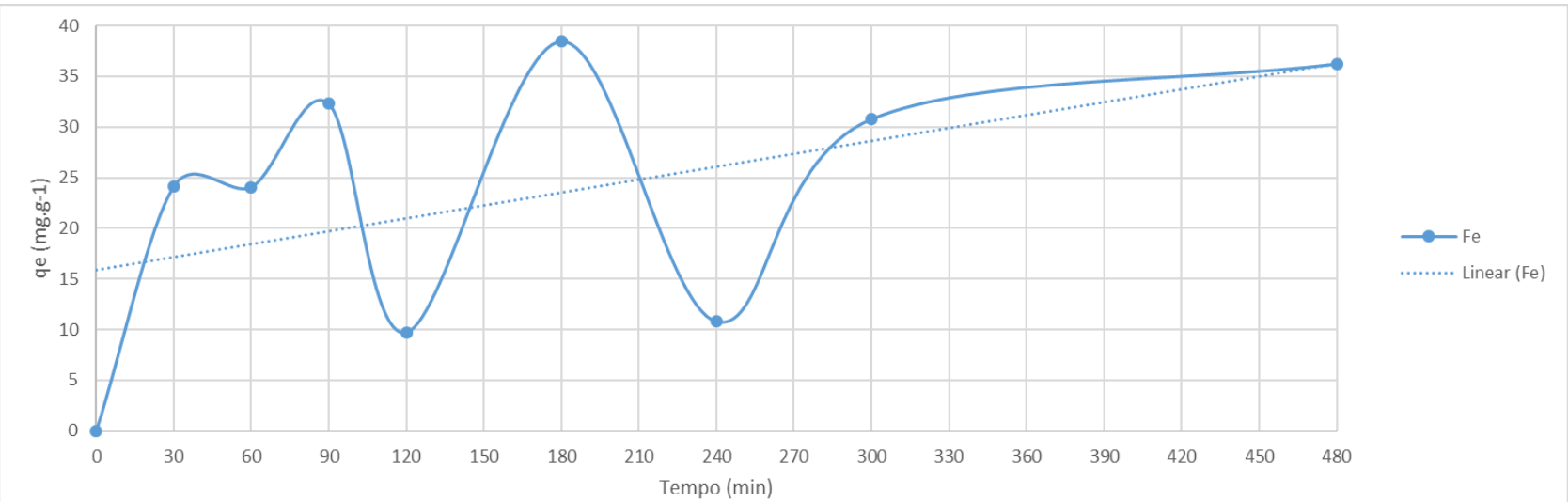

Figura 2: Variação de tempo na adsorção de ferro

A capacidade de adsorção do ferro é a maior entre todos os metais por estar presente em maior concentração, ocupando assim mais sítios ativos da resina quelante, além do fato de a carga do íon (+3) aumentar a quantidade de sítios ativos ocupados na resina.

\subsection{Influência do pH}

$\mathrm{O} \mathrm{pH}$ estudado foi entre 0.5 e 2. Nestes valores de $\mathrm{pH}$ estudados, há competição entre os íons $\mathrm{H}^{+}$e os cátions metálicos na reação de troca-iônica. A Figura 3 apresenta o comportamento do grupo funcional iminodiacetato com a variação do $\mathrm{pH}$. Como é possível observar, em $\mathrm{pH} 2.21$, a presença de $\mathrm{H}^{+}$interfere no processo de adsorção pelos cátions metálicos. Essa competição entre $\mathrm{H}^{+}$e os cátions diminui com o aumento do pH. Entretanto, devido à precipitação do ferro em pH acima de 2 não é possível realizar ensaios em pH básico (16). Desta forma, o processo de troca-iônica depende da composição da solução a ser trabalhada (13). 
<smiles>[R]CN(CC(=O)O)CC(=O)O</smiles><smiles>[R]C[NH+](CC(=O)O)[C@H](C)C(=O)O</smiles><smiles></smiles><smiles>[R]CN(CC(=O)O)[C@H]([2H])C(=O)[O-]</smiles>

Figura 3: Protonação do grupo funcional iminodiacetato (13)

Assim, era esperado maior recuperação de cobre em pH mais elevado, neste caso em pH 2, como observado na Figura 4. Em pH 0.5 foi possível recuperação de $10 \%$ do cobre, e este valor aumentou para $50 \% \mathrm{em} \mathrm{pH} 2$. O íon férrico foi o cátion com maior quantidade adsorvida em massa de íon por massa de resina $\left(26 \mathrm{mg}^{-1} \mathrm{~g}^{-1}\right)$, o que representa apenas $1.78 \%$ de todo íon férrico presente na solução, seguido pelo magnésio (20mg.g $\left.{ }^{-1}-3.73 \%\right)$ e alumínio $\left(9 \mathrm{mg} \cdot \mathrm{g}^{-1}-3.43 \%\right)$.

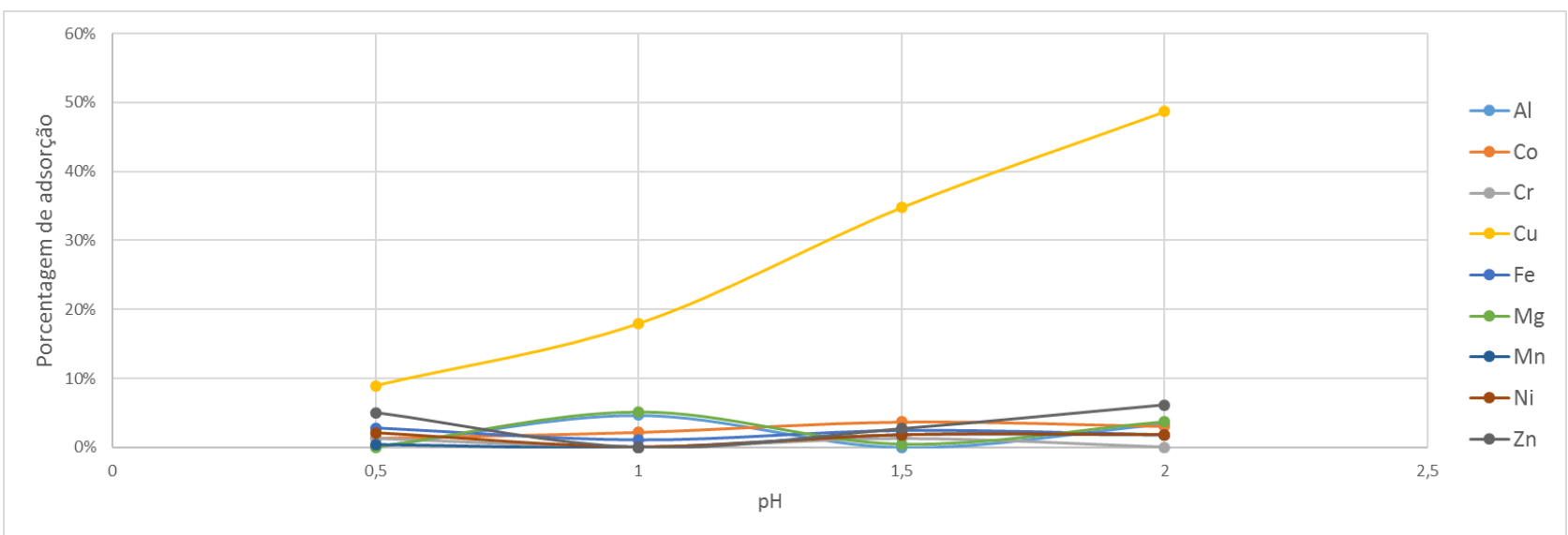

Figura 4: Porcentagem de recuperação dos metais do licor de níquel

Após o cobre, o cátion metálico que mais foi absolvido pela resina foi o zinco, com 6\%. O coeficiente de distribuição (Kd) é apresentado na Figura 5. Como é possível verificar, em pH 0.5 o valor é de $10 \mathrm{~mL} . \mathrm{g}^{-1}$ para o cobre e $80 \mathrm{~mL}^{\circ} \mathrm{g}^{-1} \mathrm{em} \mathrm{pH} 2$. 


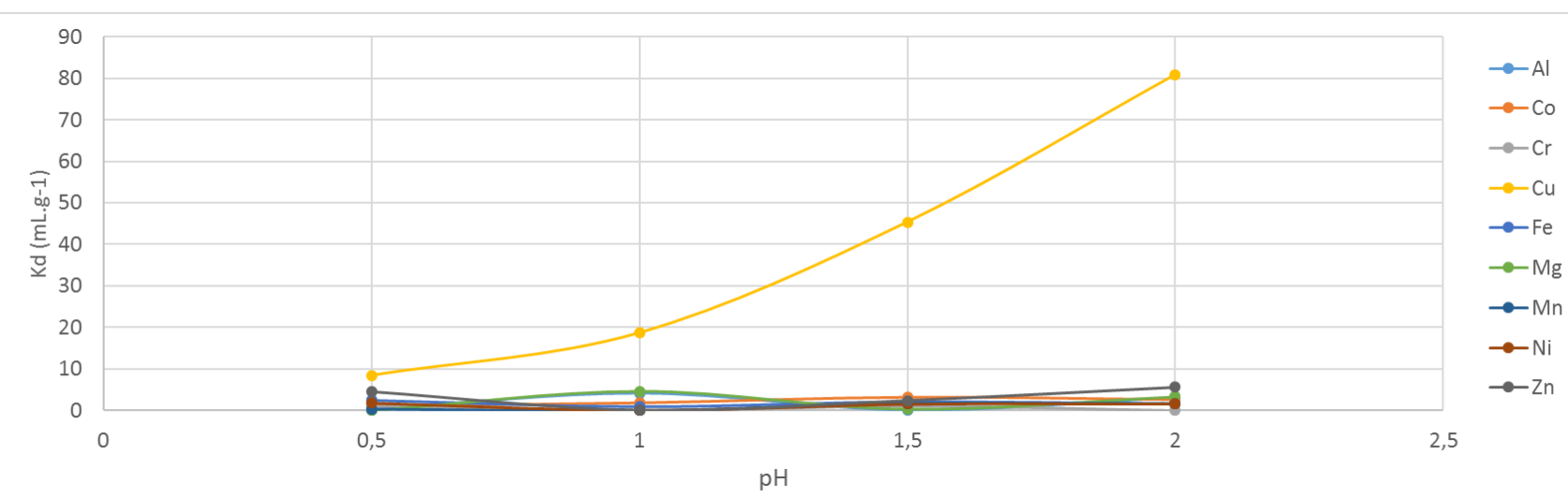

Figura 5: Coeficiente de distribuição $(\mathrm{Kd})$ da resina conforme varia $\mathrm{pH}$

O zinco, logo após o cobre, teve Kd de $4.5 \mathrm{~mL} . \mathrm{g}^{-1} \mathrm{em} \mathrm{pH} 0.5$ e $5.6 \mathrm{~mL} \cdot \mathrm{g}^{-1} \mathrm{em}$ $\mathrm{pH}$ 2. Isto posto, a resina não seguiu a seletividade esperada: $\mathrm{Fe}(\mathrm{III})>\mathrm{Cu}(\mathrm{II})>\mathrm{Ni}$ (II) $>\mathrm{Zn}(\mathrm{II})>\mathrm{Mn}(\mathrm{II})>\mathrm{Mg}(\mathrm{II})$, o que indica que há competitividade entre os cátions com o grupo funcional da resina, além de apresentar alta seletividade para o cobre frente os outros metais do licor.

\section{CONCLUSÃO}

A resina quelante Lewatit TP 207 mostrou-se específica para o cobre no processo de quelação entre os metais que compõe o licor de lixiviado de níquel. A presença de ferro impede que seja realizado ensaios em $\mathrm{pH}$ acima de 2, onde ocorreria precipitação do mesmo. Além disso, a concentração do íon férrico e do alumínio, e suas respectivas cargas (+3), fazem com que os mesmos ocupem mais sítios ativos do que os outros cátions bivalentes. Entretanto, foi possível recuperar $50 \%$ do íon presente na solução com 180min de ensaio em batelada. O uso da técnica de troca-iônica pode vir a ser bem utilizada na mineração, sobretudo para recuperação de metais de baixo teor. Outra vantagem é que não há geração de gases de efeito estufa ou resíduos no processo, que por vezes inviabilizam processos extrativos.

\section{Agradecimentos}

À Fundação de Amparo à Pesquisa do Estado de São Paulo (FAPESP - $n^{\circ}$ 2012/51871-9 e n²016/05527-5) pelo suporte financeiro.

\section{REFERÊNCIAS}

1. Crundwell FK, Moats MS, Ramachandran V, Robinson TG, Davenport WG. Extractive metallurgy of nickel, cobalt and platinum-group metals. 2011.

2. Mudd GM. Global trends and environmental issues in nickel mining: Sulfides versus laterites. Ore Geol Rev [Internet]. 2010;38(1-2):9-26. Available from: http://dx.doi.org/10.1016/j.oregeorev.2010.05.003

3. Kossoff D, Dubbin WE, Alfredsson M, Edwards SJ, Macklin MG, HudsonEdwards KA. Mine tailings dams: Characteristics, failure, environmental impacts, and remediation. Appl Geochemistry [Internet]. 2014;51:229-45. Available from: http://dx.doi.org/10.1016/j.apgeochem.2014.09.010

4. Dalvi AD, Bacon WG, Osborne RC. The Past and the Future of Nickel Laterites. PDAC 2004 Int Conv. 2004;(Figure 2):1-27.

5. Mudd GM, Jowitt SM. A Detailed Assessment of Global Nickel Resource 
Trends and Endowments. Econ Geol [Internet]. 2014 Nov 1;109(7):1813-41. Available from:

http://economicgeology.org/cgi/doi/10.2113/econgeo.109.7.1813

6. Azam S, Li Q. Tailings dam failures: A review of the last one hundred years. Geotech News. 2010;28(4):50-3.

7. Marta-Almeida M, Mendes R, Amorim FN, Cirano M, Dias JM. Fundão Dam collapse: Oceanic dispersion of River Doce after the greatest Brazilian environmental accident. Mar Pollut Bull [Internet]. 2016;112(1-2):359-64. Available from: http://linkinghub.elsevier.com/retrieve/pii/S0025326X16306038

8. Fernandes GW, Goulart FF, Ranieri BD, Coelho MS, Dales K, Boesche N, et al. Deep into the mud: ecological and socio-economic impacts of the dam breach in Mariana, Brazil. Nat Conserv [Internet]. 2016;1-11. Available from: http://linkinghub.elsevier.com/retrieve/pii/S1679007316301104

9. Fu F, Wang Q. Removal of heavy metal ions from wastewaters: A review. J Environ Manage [Internet]. 2011;92(3):407-18. Available from: http://dx.doi.org/10.1016/j.jenvman.2010.11.011

10. Jha MK, Kumari A, Panda R, Rajesh Kumar J, Yoo K, Lee JY. Review on hydrometallurgical recovery of rare earth metals. Hydrometallurgy [Internet]. 2016;161:77-101. Available from: http://dx.doi.org/10.1016/j.hydromet.2016.01.035

11. Harland CE. Ion Eschange: Theory and Practice. The Royal Society of Chemistry; 1994. 306 p.

12. Abrão A. Operações De Troca lônica. São Paulo: INSTITUTO DE PESQUISAS ENERGÉTICAS E NUCLEARES - CNEN/SP; 2014. 201 p.

13. Rudnicki $P$, Hubicki Z, Kołodyńska D. Evaluation of heavy metal ions removal from acidic waste water streams. Chem Eng J. 2014;252:362-73.

14. Zainol Z, Nicol MJ. Ion-exchange equilibria of $\mathrm{Ni2}+, \mathrm{Co} 2+, \mathrm{Mn2}+$ and $\mathrm{Mg} 2+$ with iminodiacetic acid chelating resin Amberlite IRC 748. Hydrometallurgy [Internet]. 2009;99(3-4):175-80. Available from: http://dx.doi.org/10.1016/j.hydromet.2009.08.004

15. Yu Z, Qi T, Qu J, Wang L, Chu J. Removal of $\mathrm{Ca}$ (II) and $\mathrm{Mg}$ (II) from potassium chromate solution on Amberlite IRC 748 synthetic resin by ion exchange. J Hazard Mater. 2009;167(1-3):406-12.

16. Jiménez Correa MM, Aliprandini P, Tenório JAS, Espinosa DCR. Precipitation of Metals From Liquor Obtained in Nickel Mining. Rewas 2016 Towar Mater Resour Sustain. 2016;333-8. 